BMJ Open

Diabetes

Research

\& Care

\title{
Variations of dietary intake by glycemic status and Hispanic/Latino heritage in the Hispanic Community Health Study/ Study of Latinos (HCHS/SOL)
}

\author{
Sarah S Casagrande, ${ }^{1}$ Daniela Sotres-Alvarez, ${ }^{2}$ Larissa Avilés-Santa, ${ }^{3}$ \\ Matthew J O'Brien, ${ }^{4}$ Cristina Palacios, ${ }^{5}$ Cynthia M Pérez, ${ }^{6}$ Samantha A Reina, ${ }^{7}$ \\ Xueyin Wang, ${ }^{8}$ Qibin Qi, ${ }^{8}$ Aida L Giachello, ${ }^{9}$ Rebeca A Espinoza Giacinto, ${ }^{10}$ \\ Catherine C Cowie ${ }^{11}$
}

To cite: Casagrande SS, Sotres-Alvarez D,

Avilés-Santa L, et al. Variations of dietary intake by glycemic status and Hispanic/Latino heritage in the Hispanic Community Health Study/ Study of Latinos (HCHS/SOL). BMJ Open Diab Res Care 2018;6:e000486. doi:10.1136/ bmjdrc-2017-000486

- Additional material is published online only. To view please visit the journal online (http://dx.doi.org/10.1136/ bmjdrc-2017-000486).

Received 13 October 2017 Revised 11 December 2017 Accepted 29 December 2017

Check for updates

For numbered affiliations see end of article.

Correspondence to Dr Sarah S Casagrande; scasagrande@s-3.com

\section{ABSTRACT}

Objective A healthy diet is important for diabetes prevention and control; however, few studies have assessed dietary intake among US Hispanics/Latinos, a diverse population with a significant burden of diabetes. To address this gap in the literature, we determined intake of energy, macro/micronutrients, and vitamin supplements among Hispanics/Latinos by glycemic status and heritage. Research design and methods Cross-sectional study of adults aged 18-74 years from the Hispanic Community Health Study/Study of Latinos (2008-2011) with complete baseline data on glycemic status and two 24-hour dietary recalls ( $n=13089$ ). Age-adjusted and sex-adjusted and multivariable-adjusted measures of intake were determined by glycemic status and heritage.

Results Mean age-adjusted and sex-adjusted energy intake was significantly lower among Hispanics/Latinos with diagnosed diabetes compared with those with normal glycemic status (1665 vs 1873 kcal, $P<0.001$ ). Fiber intake was higher among those with diagnosed diabetes versus normal glycemic status $(\mathrm{P}<0.01)$. Among those with diagnosed diabetes, energy intake was highest among those with Cuban heritage compared with most other heritage groups ( $\mathrm{P}<0.01$ for all, except Mexicans), but there was no difference after additional adjustment. Fiber intake was significantly lower for those of Cuban heritage (vs Dominican, Central American, and Mexican), and sodium intake was significantly higher (vs all other heritage groups) ( $P<0.01$ for all); findings were null after additional adjustment. There was no difference in supplemental intake of vitamin D, calcium, magnesium, or potassium by glycemic status.

Conclusions As part of the care of Hispanics/Latinos with diabetes, attention should be made to fiber and sodium consumption.

\section{INTRODUCTION}

Hispanics/Latinos represent $17.6 \%$ of the total US population and are more likely to have diagnosed diabetes than other ethnic groups. ${ }^{12}$ US data from 2013 to 2015 indicated that $12.1 \%$ of Hispanics reported diagnosed diabetes compared with $7.4 \%$ of non-Hispanic

\section{Significance of this study}

What is already known about this subject?

- The prevalence of diabetes among US Hispanics/ Latinos is significantly higher compared with non-Hispanic whites.

- A healthy diet is important for diabetes prevention and control; thus, diet modification is often the first line of treatment.

What are the new findings?

- Among those with diagnosed diabetes, energy and sodium intake was higher and fiber intake was lower for those with Cuban heritage compared with most other heritage groups; thus, dietary intake may be specific to heritage

How might these results change the focus of research or clinical practice?

- Given the importance of healthy diets in the self-management of diabetes, physicians should understand variation in dietary intake by Hispanic/ Latino heritage and, with this knowledge, inform patients on nutrients especially important for reducing cardiovascular disease risk.

whites. ${ }^{2}$ However, the prevalence of diabetes varies by Hispanic/Latino heritage. Baseline data (2008-2011) from the Hispanic Community Health Study/Study of Latinos (HCHS/ SOL), which included participants from four communities in the USA, estimated that the prevalence of diabetes ranged from $10.2 \%$ among those with South American heritage to $18.3 \%$ among those with Mexican heritage. ${ }^{3}$ In addition to the higher prevalence of diabetes among Hispanics/Latinos, previous work has shown that Mexican Americans may have worse control of diabetes compared with other racial/ethnic groups. In a US survey, Mexican Americans were less likely to meet 
A1c and low-density lipoprotein (LDL) cholesterol goals compared with non-Hispanic whites $(43.5 \%$ vs $52.9 \%$ for $\mathrm{A} 1 \mathrm{c}<7.0 \%$ and $47.4 \%$ vs $62.1 \%$ for $\mathrm{LDL}<100 \mathrm{mg}$ / $\mathrm{dL}$ ), and about one-third of Mexican Americans had elevated blood pressure $(\geq 140 / 90 \mathrm{~mm} \mathrm{Hg})$ compared with one-quarter of non-Hispanic whites. ${ }^{4}$ Poor control of diabetes may result in higher rates of some complications. For example, Hispanics from a national survey with diabetes have been shown to have a higher prevalence of end-stage renal disease compared with whites; however, cardiovascular disease and visual impairment have been shown to be lower in Hispanics with diabetes. ${ }^{5}$

A healthy diet is important for diabetes prevention and control; thus, diet modification is often the first line of treatment before initiating pharmacological therapy. ${ }^{6}$ Previous research from a US health survey reported that between 1982 and 2006 the percent of calories from total fat, saturated fat, and protein among Mexican Americans decreased while carbohydrate and total energy intake increased. ${ }^{7}$ Other studies using US data have shown that Hispanics have higher intakes of dietary fiber compared with other racial/ethnic groups, although consumption was still below recommendations. ${ }^{8}$ Data from the entire HCHS/SOL showed that dietary intake varied by Hispanic/Latino heritage; those with Cuban heritage had higher intakes of energy while those with Dominican heritage reported the lowest intake of energy. ${ }^{9}$ However, few studies have assessed dietary intake among Hispanics by glycemic status. Previous work assessing nutritional intake by awareness of diabetes showed that energy intake was similar among those with diagnosed and undiagnosed diabetes. In addition, awareness of diabetes was associated with lower carbohydrate and sugar intake in some Hispanic/Latino heritage groups. ${ }^{10}$ Finally, in a national study among adults with diabetes, energy intake was similar for non-Hispanic whites and Mexican Americans; intake was lower for those with diabetes compared with those without diabetes. ${ }^{11}$

There are several micronutrients that may be important for diabetes prevention and management. Some studies have shown that low vitamin $\mathrm{D}$ and calcium levels are associated with worse glycemic control while other studies have shown no association. ${ }^{12-14}$ Nevertheless, persons with diabetes are at an increased risk of fractures; thus, calcium and vitamin $\mathrm{D}$ are important for bone health in this population. ${ }^{15}$ Vitamin $\mathrm{C}$ and potassium are makers for fruits and vegetable intake since they are found in a variety of produce, and potassium has been shown to influence insulin secretion. ${ }^{16}{ }^{17}$ In some studies, magnesium deficiency has been associated with type 2 diabetes. ${ }^{18}{ }^{19}$ Despite these associations, the American Diabetes Association (ADA) does not have specific micronutrient guidelines for persons with diabetes since the scientific evidence is insufficient.

The purpose of this study was to use baseline data from the HCHS/SOL to report energy, macronutrient and micronutrient intake, and supplemental intake of key vitamins among Hispanics/Latinos by glycemic status (diagnosed diabetes, undiagnosed diabetes, pre-diabetes, and normal glucose status). In addition, intake by glycemic status was assessed by Hispanic/Latino heritage. Results from this study provide a first and comprehensive look at the diet of Hispanics/Latinos in four large US communities by glycemic status and Hispanic/Latino heritage.

\section{RESEARCH DESIGN AND METHODS}

The HCHS/SOL is a community-based cohort study of 16415 self-identified Hispanic/Latino adults aged 18-74 years from randomly selected households in four US field centers (Chicago, Illinois; Miami, Florida; Bronx, New York; San Diego, California). Baseline data were collected at a clinic visit in 2008-2011. Sample design and cohort selection have been previously described. ${ }^{20}$ Participants provided consent to participate in the research. The household-level response rate was $33.5 \%$, and of the individuals who were screened, selected, and met eligibility criteria, $41.7 \%$ were enrolled; non-response adjustment is described in the 'Statistical analysis' section.

\section{Study participants}

Participants were adults aged 18-74 years who had complete information on glycemic status and who had completed both 24-hour dietary recalls $(\mathrm{n}=13089)$. Participants self-reported sociodemographic characteristics (age, sex, Hispanic/Latino heritage, education level, years living in the USA or its territories, and household annual income); time since diagnosis of diabetes; use of insulin and oral medications; and minutes of sedentary time per day. Height and weight were measured during the examination to determine body mass index (BMI, $\left.\mathrm{kg} / \mathrm{m}^{2}\right)$.

\section{Glycemic status}

Glycemic status was defined as a four-level categorical variable: diagnosed diabetes, undiagnosed diabetes, pre-diabetes, and normal glucose status. Specifically, adults who self-reported a physician diagnosis of diabetes were classified as having diagnosed diabetes $(\mathrm{n}=1825)$. Undiagnosed diabetes was defined as having an A1c $\geq 6.5 \%$, a fasting plasma glucose (FPG) $\geq 126 \mathrm{mg}$ / $\mathrm{dL}$, or 2-hour plasma glucose from an oral glucose tolerance test $(2 \mathrm{HPG}) \geq 200 \mathrm{mg} / \mathrm{dL}$ and no physician diagnosis of diabetes $(n=1005)$; pre-diabetes was defined as having an A1c $5.7 \%$ to $<6.5 \%$, an FPG 100 to $<126 \mathrm{mg}$ / $\mathrm{dL}$, or a $2 \mathrm{HPG} 140$ to $<200 \mathrm{mg} / \mathrm{dL}$ and no physician diagnosis of diabetes $(n=5261)$; normal glucose level was defined as having an A1c $<5.7 \%$ and an FPG $<100 \mathrm{mg} / \mathrm{dL}$ and $2 \mathrm{HPG}<140 \mathrm{mg} / \mathrm{dL} \quad(\mathrm{n}=4998)$. Medication use was not included as part of the definition of glycemic status since roughly $15 \%$ of adults with diabetes do not take any glycemic medications and adults with pre-diabetes are often using oral diabetes medications. 


\section{Dietary intake}

Participants were asked to report all foods and beverages consumed in the past 24 hours during the baseline interview. ${ }^{92}$ A second 24-hour dietary recall was performed by telephone approximately 30 days after the interview. Interviews were conducted with the Nutrition Data System for Research software V.11 developed by the Nutrition Coordination Center at the University of Minnesota. Dietary intake was determined by averaging the two 24-hour dietary recalls; $83 \%$ of participants in the HCHS/ SOL had both dietary recalls $(n=14160)$. Interviews were conducted using the computerized multiple-pass method where interviewers collect a quick list of all foods and beverages consumed in the previous day; probe for foods potentially forgotten in the quick list; collect the time and occasion for each food; collect a detailed description of the food, the amount, and additions (eg, salt, oil) and review the 24-hour day; and conduct a final probe for anything else consumed. Diet assessment was performed using the Nutrition Data System for Research software developed by the Nutrition Coordinating Center at the University of Minnesota; the database included common foods, brand name foods, and common Hispanic/Latino foods. Recalls that were deemed unreliable by the interviewer or where energy intake was below the 1 st or above the 99 th percentile by sex were excluded $(n=617,1.9 \%)$. Our analysis reports on total energy intake; percent of calories from carbohydrates, protein, total fat, saturated fat, polyunsaturated fat, and monounsaturated fat; fiber; cholesterol; alcohol; sodium; vitamin D; calcium; magnesium, and potassium. In addition, participants reported any supplements taken in the past 24 hours. Our analysis reports on mean supplemental intake of vitamin D $(n=3056)$, calcium $(n=3411)$, magnesium $(n=2569)$, and potassium $(n=2024)$ among those who reported taking the supplement in the past 30 days.

Participants additionally reported on whether they ate $^{1}$ mainly Hispanic foods or mostly Hispanic foods and some American food, ${ }^{2}$ equal amounts of both Hispanic and American foods, or ${ }^{3}$ mostly American food and some Hispanic food or mainly American food. Participants reported the frequency of eating at fast-food restaurants, including Latino and Chinese food, as never, less than once a week, 1-2 times per week, 3-4 times per week, or $\geq 5$ times per week.

\section{Statistical analysis}

The percent distribution (\%, SE) of study participant characteristics was determined by four-level glycemic status (diagnosed diabetes, undiagnosed diabetes, pre-diabetes, and normal glucose status). Dietary intake (mean, SE) was determined by glycemic status and was age-adjusted and sex-adjusted to adults with diagnosed diabetes in the HCHS/SOL. Adjusted estimates are presented to allow for comparisons across glycemic status groups without concern for differences in the age (age groups 18-44, 45-64, $\geq 65$ years) and sex distributions. In addition, dietary intake was determined by Hispanic/Latino heritage and adjusted for demographic and behavioral factors, including education level, years living in the USA, field center, BMI, and sedentary time. Additional adjustment was conducted to understand whether differences in heritage remained after controlling for demographic and behavioral factors. Regression analysis was used to determine whether there was an interaction between glycemic status and Hispanic/Latino heritage while adjusting for covariates. Descriptive statistics were used to compare eating behavior characteristics (Hispanic vs American foods and fast-food restaurant use) by glycemic status and heritage. All statistical analyses used sample weights to account for the HCHS/SOL sampling design using SUDAAN (SUDAAN User's Manual, Release 9.2, 2008; Research Triangle Institute). The HCHS/SOL sampling weights are a product of a base weight (reciprocal of the probability of selection) and three adjustments: (1) non-response adjustments made relative to the sampling frame, (2) trimming to handle extreme values, and (3) calibration of weights to the 2010 U.S. Census target population according to age, sex, and Hispanic/ Latino background.

\section{RESULTS}

\section{Participant characteristics}

Among Hispanic/Latino adults with diagnosed diabetes, $52.5 \%$ were aged $45-64$ years compared with $18.2 \%$ among those with normal glucose levels $(\mathrm{P}<0.001)$ (online supplemental table 1). Among adults with diagnosed diabetes, the percentage of Mexican heritage was the highest $(35.9 \%$ ) followed by Puerto Rican heritage (21.8\%) and Cuban heritage $(20.2 \%)$; Mexican heritage was also the highest percentage for the other glycemic status groups. Compared with adults with normal glucose levels, fewer Hispanic/ Latino adults with diagnosed diabetes had a high school diploma/General Equivalency Diploma (GED) (68.2\% vs $88.2 \%)$ and more had a household income $\leq \$ 20000(54.6 \%$ vs $42.4 \%$ ) ( $\mathrm{P}<0.001$ for both). Fewer adults with diagnosed diabetes had lived in the USA $\leq 10$ years $(18.3 \%)$ compared with those with normal glucose levels $(37.2 \%, \mathrm{P}<0.001)$. Mean BMI was higher for those with diagnosed diabetes compared with those with normal glucose levels $(31.9 \mathrm{vs}$ $\left.27.7 \mathrm{~kg} / \mathrm{m}^{2}\right) \quad(\mathrm{P}<0.001)$. Among those with diagnosed diabetes, a large percentage had a duration of diabetes $<5$ years $(43.4 \%)$ and most were taking oral diabetes medications $(81.3 \%)$.

\section{Dietary intake by glycemic status}

Mean age-adjusted and sex-adjusted energy intake among Hispanic/Latino adults with diagnosed diabetes was 1665 $\mathrm{kcal} /$ day with $51.4 \%$ of calories from carbohydrates, $18.3 \%$ from protein, and $31.3 \%$ from total fat (table 1 ).

Age-adjusted and sex-adjusted energy intake was 130-208 kcal/day lower $(\mathrm{P}<0.001)$ for those with diagnosed diabetes compared with all other glycemic status groups, but the composition of macronutrients was similar by glycemic status. 
Table 1 Age-sex adjusted mean (SE) dietary intake of energy, macronutrients, and micronutrients by glycemic status, Hispanic Community Health Study/Study of Latinos (HCHS/SOL) 2008-2011

\begin{tabular}{|c|c|c|c|c|c|}
\hline & Guidelinesł & $\begin{array}{l}\text { Diagnosed } \\
\text { diabetes }(n=1825)\end{array}$ & $\begin{array}{l}\text { Undiagnosed } \\
\text { diabetes }(n=1005)\end{array}$ & $\begin{array}{l}\text { Pre-diabetes } \\
(n=5261)\end{array}$ & $\begin{array}{l}\text { Normal glucose } \\
\text { levels }(n=4998)\end{array}$ \\
\hline Total calories (kcal) & & $1665(21.5)$ & $1795(32.0)^{\star}$ & $1816(15.8)^{\star}$ & $1873(21.5)^{*}$ \\
\hline \multicolumn{6}{|l|}{ Percent of calories from } \\
\hline Carbohydrates (\%) & $45-65$ & $51.4(0.35)$ & $53.2(0.37)^{\star}$ & $53.3(0.24)^{\star}$ & $52.9(0.28)^{\star}$ \\
\hline Protein (\%) & $10-35$ & $18.3(0.15)$ & $17.1(0.19)^{\star}$ & $16.8(0.10)^{\star}$ & $16.5(0.13)^{\star}$ \\
\hline Total fat (\%) & $20-35$ & $31.3(0.27)$ & $30.2(0.32)$ & $30.3(0.20)^{\star}$ & $30.6(0.24)$ \\
\hline Saturated fat (\%) & $<10$ & $10.1(0.11)$ & $9.9(0.16)$ & $9.9(0.09)$ & $9.9(0.11)$ \\
\hline Polyunsaturated fat (\%) & & $6.8(0.09)$ & $6.4(0.12)$ & $6.5(0.06)^{\star}$ & $6.8(0.10)$ \\
\hline Monounsaturated fat (\%) & & $11.6(0.14)$ & $11.2(0.14)$ & $11.2(0.09)$ & $11.3(0.10)$ \\
\hline Fiber (g/1000 kcal) & 14 & $11.1(0.18)$ & $10.7(0.27)$ & $9.9(0.15)^{\star}$ & $10.0(0.17)^{\star}$ \\
\hline Cholesterol (mg) & $<300$ & $251(5.4)$ & $247(6.8)$ & $250(3.7)$ & $244(4.1)$ \\
\hline Sodium (mg) & 2300 & $2963(56.5)$ & 3097 (68.1) & $3016(38.3)$ & $3107(45.1)$ \\
\hline Alcohol (g) & $14-28$ & $1.4(0.22)$ & $3.0(0.46)^{\star}$ & $3.1(0.23)^{\star}$ & $4.4(0.45)^{\star}$ \\
\hline \multicolumn{6}{|l|}{ Dietary sources } \\
\hline Vitamin D $(\mu \mathrm{g})$ & $5-15$ & $5.4(0.16)$ & $4.9(0.16)$ & $5.2(0.14)$ & $5.1(0.14)$ \\
\hline Calcium (mg) & $1000-1200$ & $775(13.8)$ & $741(15.5)$ & $767(10.5)$ & $787(14.1)$ \\
\hline Magnesium (mg) & $310-420$ & $279(4.4)$ & $286(5.6)$ & $281(5.6)$ & $293(4.6)$ \\
\hline Potassium (mg) & 4700 & $2426(34.2)$ & $2475(47.0)$ & $2442(21.2)$ & $2546(38.6)$ \\
\hline \multicolumn{6}{|l|}{ Supplemental sources: $†$} \\
\hline Vitamin D $(\mu \mathrm{g})$ & & $18.3(5.63)$ & $9.6(0.55)$ & $11.2(0.77)$ & $9.5(0.66)$ \\
\hline Calcium (mg) & & $392(20.6)$ & $364(21.9)$ & $361(15.8)$ & $346(19.4)$ \\
\hline Magnesium (mg) & & $84.1(5.54)$ & $76.5(7.40)$ & $89.0(4.83)$ & $89.5(9.50)$ \\
\hline Potassium (mg) & & $67.7(4.21)$ & 63.5 (4.34) & $68.4(5.87)$ & 61.5 (2.34) \\
\hline
\end{tabular}

Age and sex adjusted to adults with diagnosed diabetes in the HCHS/SOL (20.4\% age $20-44$ years, $52.5 \%$ age $45-64$ years, $27.1 \%$ age 65-74 years).

${ }^{*} \mathrm{P}<0.01$ versus diagnosed diabetes.

†Among those taking the supplement of interest; vitamin $D(n=3056)$, calcium $(n=3411)$, magnesium $(n=2569)$, and potassium ( $n=2024)$. $\ddagger$ Guidelines are the healthy eating guidelines for most Americans by the Office of Disease Prevention and Promotion. ${ }^{723}$ Recommendations may change depending on health status, age, or activity level.

Among adults with diagnosed diabetes, age-adjusted and sex-adjusted intake of fiber was $11.1 \mathrm{~g} / 1000 \mathrm{kcal}$, which was $1.1 \mathrm{~g} / \mathrm{kcal}$ higher compared with those with normal glucose levels $(\mathrm{P}<0.001)$; sodium intake $(2963 \mathrm{mg}$ ) was also high but similar by glycemic status. Intake of cholesterol, vitamin $\mathrm{D}$, calcium, magnesium, and potassium from food sources was also similar by glycemic status. Alcohol intake was lowest for those with diagnosed diabetes $(\mathrm{P}<0.01$ vs all other glycemic status groups). Among those who took vitamin D supplements (20.4\%), vitamin D supplementation was higher, but not significantly, among those with diagnosed diabetes (18.3 vs $9.5 \mu \mathrm{g}$ among those with normal glucose levels). There was no difference in supplemental intake of calcium, magnesium, or potassium by glycemic status.

\section{Dietary intake by Hispanic/Latino heritage and glycemic} status

Mean age-adjusted and sex-adjusted energy intake among adults with diagnosed diabetes was significantly higher among those with Cuban heritage (1806 kcal) compared with those with Dominican, Central American, Puerto Rican, or South American heritage $(\mathrm{P}<0.01$ for all) (figure 1 and online supplemental table 2). After adjustment for education level, years living in the USA, BMI, sedentary time, and field center, mean energy intake was similar by Hispanic/Latino heritage. Results were similar among those with undiagnosed diabetes, pre-diabetes, and those with normal glucose levels. There was no interaction between glycemic status and heritage in total energy intake.

Among those with diagnosed diabetes, there were few differences in the percent of calories from carbohydrates, protein, total fat, or saturated fat by Hispanic/Latino heritage (figure 2 and supplemental tables 3-6). Among adults with pre-diabetes, those with Cuban heritage had a lower percent of calories from carbohydrates and a higher intake of percent of calories from total fat, saturated fat, and monounsaturated fat compared with those 


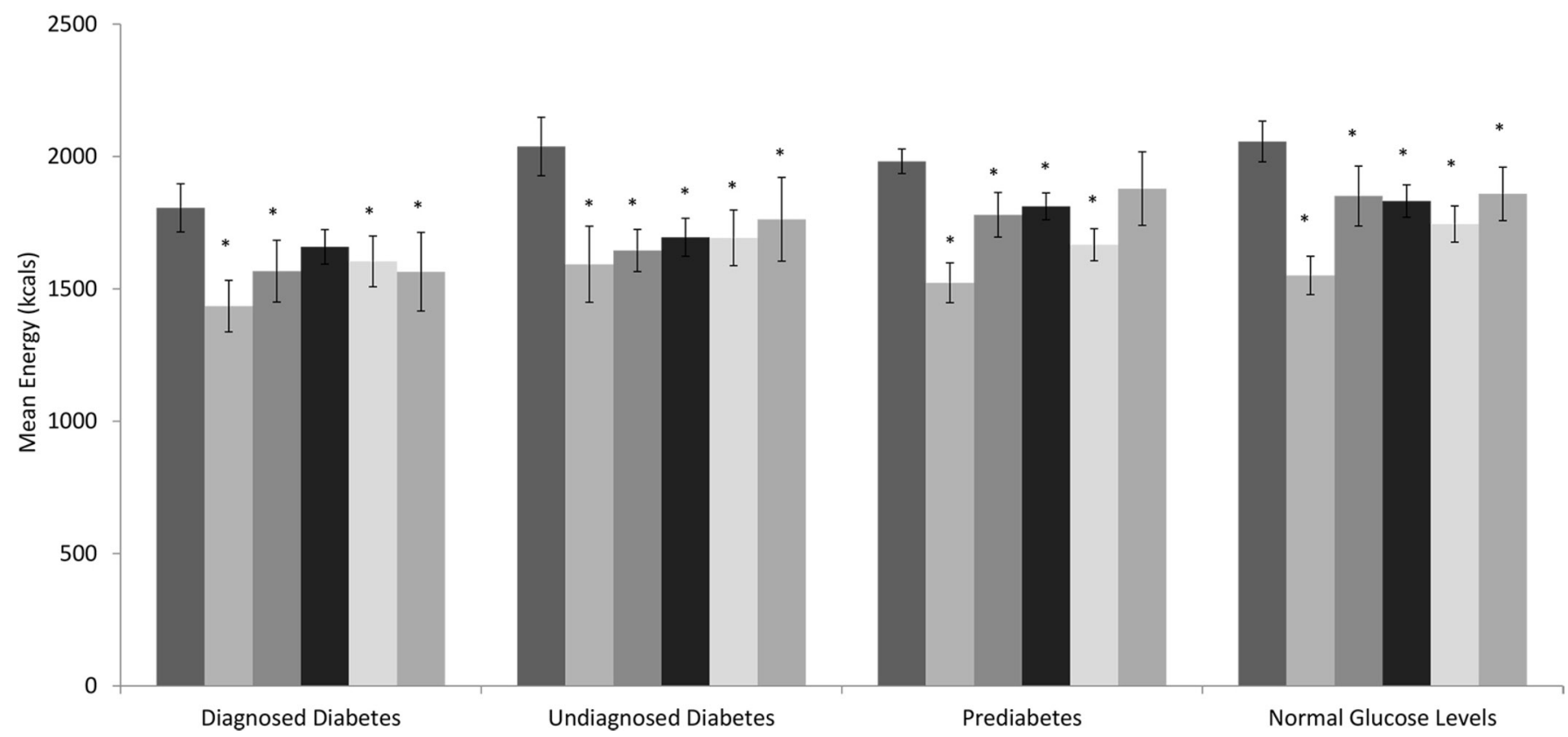

Figure 1 Age-adjusted and sex-adjusted intake of energy (kcal/day) by glycemic status and Hispanic/Latino heritage, Hispanic Community Health Study/Study of Latinos 2008-2011.

with Dominican or Central American heritage but these associations diminished after additional adjustment. Among those with diagnosed diabetes, intake of monounsaturated fat was highest for adults with Cuban heritage compared with those with Dominican, Central American, Puerto Rican, or South American heritage $(\mathrm{P}<0.01$ for all); however, these differences did not remain after additional adjustment (online supplemental table 7). There was no interaction between glycemic status and heritage for these macronutrients.
Among those with diagnosed diabetes, age-adjusted and sex-adjusted mean fiber intake was significantly lower for adults with Cuban heritage $(9.7 \mathrm{~g} / 1000 \mathrm{kcal})$ compared with those with Dominican $(11.4 \mathrm{~g} / 1000 \mathrm{kcal})$, Central American (11.4g/1000 kcal), or Mexican (13.4g/1000 kcal) heritage $(\mathrm{P}<0.01$ for all) (online supplemental table 8). These differences became non-significant after additional adjustment. Similar differences by heritage were seen among those with undiagnosed diabetes, pre-diabetes, and normal glucose levels but associations

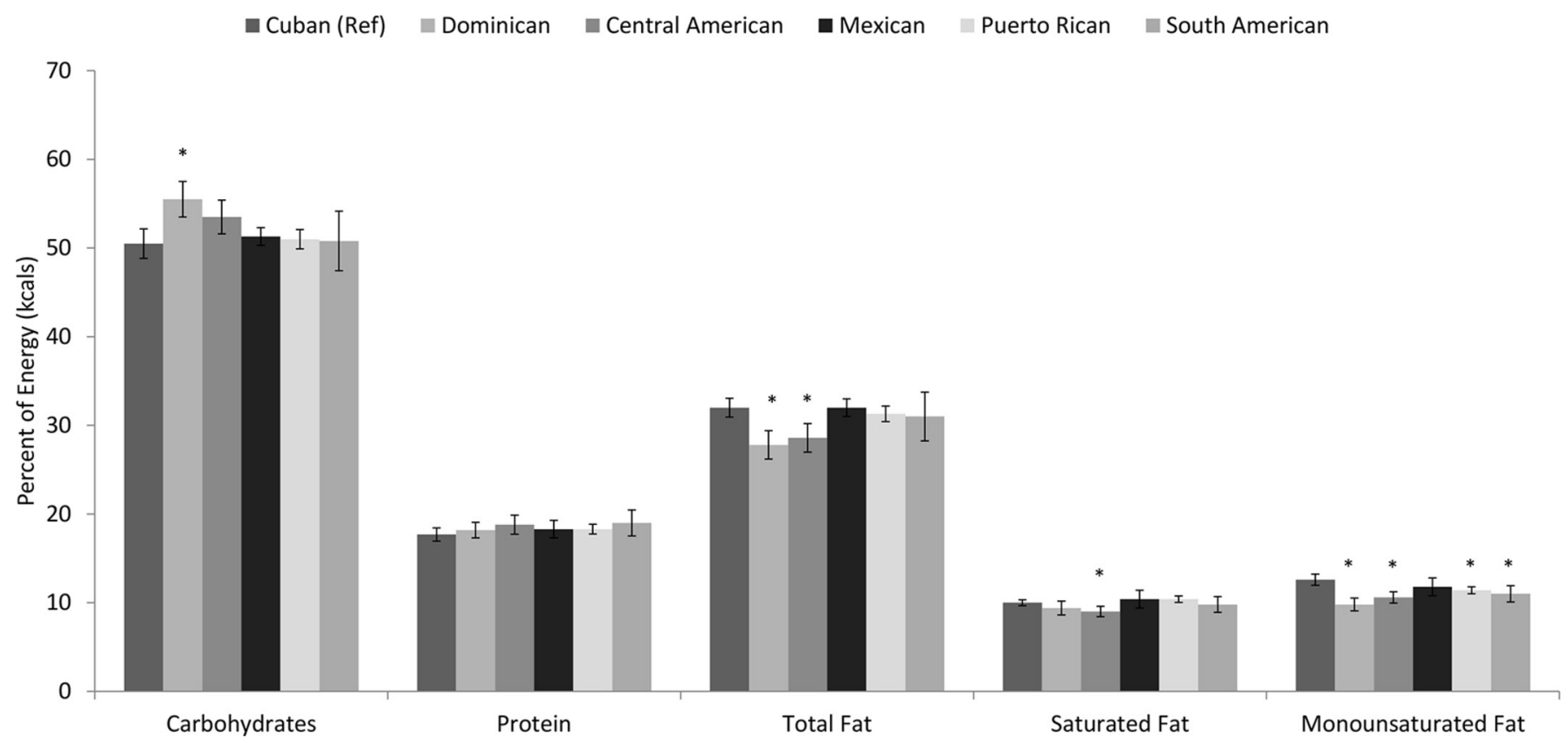

Figure 2 Age-adjusted and sex-adjusted percent of calories from macronutrients among adults with diagnosed diabetes by Hispanic/Latino heritage, Hispanic Community Health Study/Study of Latinos 2008-2011. 
remained after adjustment. There was no interaction between glycemic status and heritage in fiber intake.

Among adults with diagnosed diabetes, there were few differences in age-adjusted and sex-adjusted cholesterol intake by heritage, ranging from $201 \mathrm{mg}$ for those with Dominican heritage to $264 \mathrm{mg}$ for those with Puerto Rican heritage (online supplemental table 9). Sodium intake was highest for adults with Cuban heritage compared with all other heritage groups, regardless of glycemic status (online supplemental table 10). There were few differences in alcohol, vitamin $\mathrm{D}$, calcium, or potassium intake by heritage, regardless of glycemic status; magnesium intake was higher for those with Cuban heritage compared with those with Dominican, Central American, or Puerto Rican heritage, regardless of glycemic status but these associations diminished after adjustment (online supplemental tables 11-15).

There were few differences in supplemental intake of vitamins by heritage (data not shown). Estimates for supplemental vitamin $\mathrm{D}$ intake among those with diagnosed diabetes taking a supplement $(\mathrm{n}=522)$ were unreliable to report by heritage. Supplemental intake of calcium, magnesium, and potassium was similar by heritage for all glycemic status groups.

\section{Hispanic foods by Hispanic/Latino heritage and glycemic status}

Overall, the prevalence of consuming mainly or mostly Hispanic foods was significantly higher for those with Cuban heritage compared with those with Mexican or Puerto Rican heritage, regardless of glycemic status ( $\mathrm{P}<0.01$ for all) (figure 3$)$. Among those with diagnosed diabetes, $91.2 \%$ of those with Cuban heritage reported consuming mainly Hispanic foods compared with $74.3 \%$ of those with Mexican heritage, $63.5 \%$ of those with
Puerto Rican heritage, and $73.7 \%$ of those with South American heritage $(\mathrm{P}<0.001$ for all $)$. Among those with diagnosed diabetes, $59.5 \%$ of those with Cuban heritage reported never eating fast food compared with $39.2 \%$ of those with Dominican heritage, $41.7 \%$ of those with Central American heritage, 20.8\% of those with Mexican heritage, and $30.1 \%$ of those with Puerto Rican heritage ( $\mathrm{P}<0.01$ for all) (data not shown).

\section{CONCLUSIONS}

The ADA recommends a healthy, nutrient-dense, and balanced diet as a central component of diabetes prevention and self-management efforts; these recommendations are similar and often refer to the healthy eating guidelines for most Americans by the Office of Disease Prevention and Promotion. ${ }^{622}$ We found that energy intake was on average 130-208 kcal/day lower for US Hispanic/Latino adults with diagnosed diabetes compared with those without diagnosed diabetes, but macronutrient composition was similar by glycemic status. The percent of saturated fat approximates the recommended maximum intake of $10 \%$ of total calories. There were few other differences in dietary intake by glycemic status among Hispanics/Latinos. Fiber intake was higher among Hispanics/Latinos with diabetes compared with those with normal glucose levels; however, intake was well below the recommendations of $14 \mathrm{~g} / 1000 \mathrm{kcal}$. Sodium intake was elevated and above the recommended limit of $2300 \mathrm{mg} /$ day, regardless of glycemic status. Cholesterol intake was similar by glycemic status and fell within the general guidelines of $<300 \mathrm{mg} /$ day; however, Hispanics/ Latinos with diagnosed diabetes consumed on average $251 \mathrm{mg} /$ day, which is above the recommended limit of $200 \mathrm{mg} /$ day for adults at high risk for cardiovascular 
disease, such as those with diabetes. Calcium intake was well below recommendations of $1000-1200 \mathrm{mg} /$ day, with intake at $775 \mathrm{mg}$ for those with diagnosed diabetes; adults with diabetes are at a higher risk of bone fracture; thus, calcium is an important nutrient for those with diabetes. ${ }^{23}{ }^{24}$ Vitamin D intake was low, regardless of glycemic status, which is also crucial for bone health. Finally, magnesium and potassium intakes were below recommendations, regardless of glycemic status; magnesium deficiency has been associated with diabetes, and potassium is a marker for fruit and vegetable intake. ${ }^{17-19}$

Among adults with diagnosed diabetes, those with Cuban heritage had higher energy intake compared with all other Hispanic/Latino heritage groups with the exception of those with Mexican heritage. However, there were no differences in energy intake by heritage after adjusting for sociodemographic factors, field center, BMI, and sedentary time. Results also showed that those with Cuban heritage more often consumed traditionally Hispanic foods and were less likely to eat fast food compared with most other Hispanic/Latino heritage groups; higher energy consumption among Cubans may be related to cooking practices at home. Further study is needed to examine these associations and their impact on diabetes risk. Macronutrient intake was relatively similar by heritage among those with diagnosed diabetes. However, those with Cuban heritage had significantly higher intakes of monounsaturated fat, which is hearthealthy, compared with most other Hispanic/Latino heritage groups. Among those with diagnosed diabetes, fiber was significantly lower and sodium was significantly higher for those with Cuban heritage compared with other heritage groups; again, these associations were not seen after adjustment.

Although Hispanics/Latinos with diagnosed diabetes consumed significantly less energy compared with those without diabetes, when the results were stratified by Hispanic/Latino heritage there was little difference in consumption by glycemic status. The finding of no significant interaction between glycemic status and Hispanic/ Latino heritage was shown for all dietary outcomes. Thus, dietary intake may be specific to heritage, which has been shown previously ${ }^{9}$; but glycemic status has seemingly little influence on differences in dietary intake across Hispanic/Latino groups. Given the importance of healthy diets in the self-management and prevention of diabetes, physicians and patients should scrutinize dietary intake with specific attention to nutrients especially important for cardiovascular disease risk, including saturated fat, fiber, sodium, calcium, and vitamin D intakes.

Compared with a national sample of non-Hispanic white and black adults with diabetes in 2007-2010, Hispanics/Latinos with diagnosed diabetes from the HCHS/SOL consumed fewer calories, less saturated fat, more dietary fiber, and less sodium ${ }^{11}$; however, dietary fiber and sodium intake among Hispanics/Latinos were still not within recommendations. Given that US Hispanics have a higher prevalence of diabetes and US
Hispanics with diabetes are less likely to meet A1c and LDL-cholesterol targets compared with non-Hispanic whites, it is unclear what role dietary intake has with these outcomes. ${ }^{24}$ Future studies should investigate how dietary intake in Hispanics/Latinos with several different heritages may influence diabetes control and prevention of complications. Results from the current study showed variation in frequency of eating traditionally Hispanic foods and of eating fast food by heritage. Use of national data that do not distinguish Hispanic/Latino heritage may not be appropriate for practitioners or for public health messages.

One strength of this study was the use of a large and diverse sample of Hispanics/Latinos living in the USA that included several Hispanic/Latino heritage groups. Two 24-hour dietary recalls were used to determine dietary intake, which is a reliable method to determine average intake. ${ }^{25}$ However, dietary intake was self-reported, which could result in measurement error or bias; individuals who are overweight may under-report intake. ${ }^{21}$ Standardized clinical measures of glycemia were used to determine glycemic status; however, a second follow-up measure of glycemia was not available to confirm glycemic status in the HCHS/SOL, potentially causing non-differential misclassification of glycemic status. In addition, we could not distinguish type 1 and type 2 diabetes but, as recommended by the ADA, all persons with diabetes should be following a healthy diet. Although the HCHS/SOL is a large representative study of the communities surveyed with good representation of the various Hispanic/Latino heritage groups, estimates cannot be generalized to the entire US Hispanic/Latino population. Nevertheless, the four field centers are among the largest US metropolitan areas with Hispanic/Latino population or with the largest percent of the specific heritage group (eg, Cubans in Miami).

In large samples of Hispanic/Latinos living in the USA, those with diagnosed diabetes are generally adhering to the ADA's dietary recommendations. However, fiber intake should increase and, among those with or at risk for cardiovascular disease, cholesterol intake should decrease. These results are the first to examine dietary intake by glycemic status and Hispanic/Latino heritage in this high-risk population group. Physicians and public health officials should consider these results when developing culturally specific nutrition programs. Future research that aims to understand the behavioral differences in dietary intake by Hispanic/Latino heritage may give insight into reaching these groups most effectively for better disease control and prevention.

\section{Author affiliations}

${ }^{1}$ Social \& Scientific Systems, Inc., Silver Spring, Maryland, USA

${ }^{2}$ University of North Carolina, Chapel Hill, North Carolina, USA

${ }^{3}$ National Heart, Lung, and Blood Institute, Bethesda, Maryland, USA

${ }^{4}$ General Internal Medicine, Northwestern University, Chicago, Illinois, USA

${ }^{5}$ Florida International University, Miami, Florida, USA

${ }^{6}$ School of Public Health, University of Puerto Rico, Puerto Rico, USA

${ }^{7}$ University of Miami, Coral Gables, Florida, USA 
${ }^{8}$ Albert Einstein College of Medicine, Bronx, New York, USA

${ }^{9}$ Department of Preventive Medicine, Northwestern University, Chicago, Illinois, USA

${ }^{10}$ University of California, San Diego, California, USA

${ }^{11}$ National Institute of Diabetes and Digestive and Kidney Diseases, Bethesda, Maryland, USA

Contributors SSC designed the research, analyzed the data, wrote the paper, and had primary responsibility for final content. DSA and MLA-S reviewed the analysis, and edited and finalized the manuscript. MJO, CP, CMP, SAR, XW, ALG, and RAEG edited and finalized the manuscript. CCC designed the research, and edited and finalized the manuscript.

Funding This work was supported by contracts from the National Heart, Lung, and Blood Institute (NHLBI) to the University of North Carolina (N01-HC65233), University of Miami (N01-HC65234), Albert Einstein College of Medicine (N01HC65235), Northwestern University (N01-HC65236), and San Diego State University (N01-HC65237). The following NIH Institutes/Offices collaborated and co-funded the first phase of the study: the National Institute on Minority Health and Health Disparities; the National Institute on Deafness and Other Communication Disorders; the National Institute of Dental and Craniofacial Research; the National Institute of Diabetes, Digestive and Kidney Diseases; the National Institute of Neurological Disorders and Stroke; and the NIH Office of Dietary Supplements. This manuscript was supported by a contract (GS10F0381L) from the National Institute of Diabetes and Digestive and Kidney Diseases (NIDDK).

Disclaimer The views expressed in this article are those of the authors and do not necessarily represent the views of the National Heart, Lung, and Blood Institute; the National Institute of Diabetes and Digestive and Kidney Diseases; the National Institutes of Health; or the U.S. Department of Health and Human Services.

Competing interests None declared.

Patient consent Obtained.

Provenance and peer review Not commissioned; externally peer reviewed. Data sharing statement Data from HCHS/SOL can be accessed by submitting proposals for manuscripts through the HCHS/SOL website, http://www2.cscc.unc. edu/hchs/.

Open Access This is an Open Access article distributed in accordance with the Creative Commons Attribution Non Commercial (CC BY-NC 4.0) license, which permits others to distribute, remix, adapt, build upon this work non-commercially, and license their derivative works on different terms, provided the original work is properly cited and the use is non-commercial. See: http://creativecommons.org/ licenses/by-nc/4.0/

(C) Article author(s) (or their employer(s) unless otherwise stated in the text of the article) 2018. All rights reserved. No commercial use is permitted unless otherwise expressly granted.

\section{REFERENCES}

1. U.S. Census Bureau. The hispanic population: 2015. In: U.S. Department of Commerce. ed. Suitland, MD: Economics and Statistics Administration, 2015.

2. National Center for Chronic Disease Prevention and Health Promotion. National diabetes statistics report, 2017. In: U.S. Department of Health and Human Services. Atlanta, GA: Centers for Disease Control and Prevention, 2017.

3. Schneiderman N, Llabre M, Cowie CC, et al. Prevalence of diabetes among Hispanics/Latinos from diverse backgrounds: the Hispanic Community Health Study/Study of Latinos (HCHS/SOL). Diabetes Care 2014;37:2233-9.
4. Stark Casagrande S, Fradkin JE, Saydah SH, et al. The prevalence of meeting $\mathrm{A} 1 \mathrm{C}$, blood pressure, and LDL goals among people with diabetes, 1988-2010. Diabetes Care 2013;36:2271-9.

5. Saydah SH, Fradkin J, Cowie CC. Poor control of risk factors for vascular disease among adults with previously diagnosed diabetes. JAMA 2004;291:335-42.

6. American Diabetes Association. Standards of medical care in diabetes-2011. Diabetes Care 2011;34(Suppl 1):S11-61.

7. Fryar CD, Wright JD, Eberhardt MS, et al. Trends in nutrient intakes and chronic health conditions among Mexican-American adults, a 25-year profile: United States, 1982-2006. Natl Health Stat Report 2012;50:1-20.

8. Storey M, Anderson P. Income and race/ethnicity influence dietary fiber intake and vegetable consumption. Nutr Res 2014;34:844-50.

9. Siega-Riz AM, Sotres-Alvarez D, Ayala GX, et al. Food-group and nutrient-density intakes by Hispanic and Latino backgrounds in the Hispanic Community Health Study/Study of Latinos. Am J Clin Nutr 2014;99:1487-98.

10. Wang $X$, Jung $M$, Mossavar-Rahmani $Y$, et al. macronutrient intake, diagnosis status, and glycemic control among US Hispanics/Latinos with diabetes. J Clin Endocrinol Metab 2016;101:1856-64.

11. Casagrande SS, Cowie CC. Trends in dietary intake among adults with type 2 diabetes: NHANES 1988-2012. J Hum Nutr Diet 2017;30:479-89.

12. Pittas AG, Lau J, Hu FB, et al. The role of vitamin D and calcium in type 2 diabetes. a systematic review and meta-analysis. J Clin Endocrinol Metab 2007;92:2017-29.

13. Krul-Poel YH, Westra S, ten Boekel E, et al. Effect of Vitamin D supplementation on glycemic control in patients with type 2 diabetes (sunny trial): a randomized placebo-controlled trial. Diabetes Care 2015;38:1420-6.

14. Pittas AG, Dawson-Hughes B, Sheehan PR, et al. Rationale and design of the Vitamin $D$ and type 2 Diabetes (D2d) study: a diabetes prevention trial. Diabetes Care 2014;37:3227-34.

15. Shanbhogue VV, Mitchell DM, Rosen CJ, et al. Type 2 diabetes and the skeleton: new insights into sweet bones. Lancet Diabetes Endocrinol 2016;4:159-73.

16. U.S National Library of Medicine. Vitamin C. Bethesda, MD, 2015. https://www.nlm.nih.gov/medlineplus/vitaminc.html. (accessed 5 Nov 2015)

17. Chatterjee R, Yeh HC, Shafi T, et al. Serum and dietary potassium and risk of incident type 2 diabetes mellitus: The Atherosclerosis Risk in Communities (ARIC) study. Arch Intern Med 2010;170:1745-51.

18. Chaudhary DP, Sharma R, Bansal DD. Implications of magnesium deficiency in type 2 diabetes: a review. Biol Trace Elem Res 2010;134:119-29.

19. Tosiello L, Hypomagnesemia TL. Hypomagnesemia and diabetes mellitus. A review of clinical implications. Arch Intern Med 1996;156:1143-8.

20. Lavange LM, Kalsbeek WD, Sorlie PD, et al. Sample design and cohort selection in the Hispanic Community Health Study/Study of Latinos. Ann Epidemiol 2010;20:642-9.

21. Moshfegh AJ, Rhodes DG, Baer DJ, et al. The US Department of Agriculture Automated Multiple-Pass Method reduces bias in the collection of energy intakes. Am J Clin Nutr 2008;88:324-32.

22. U.S. Department of Agriculture and U.S. Department of Health and Human Services. Dietary guidelines for americans, 2010. 7th Edition. Washington, D.C: U.S. Government Printing Office, 2010.

23. Meier C, Schwartz AV, Egger A, et al. Effects of diabetes drugs on the skeleton. Bone 2016;82:93-100.

24. Yamamoto M. Insights into bone fragility in diabetes: the crucial role of bone quality on skeletal strength. Endocr $J$ 2015;62:299-308.

25. Basiotis PP, Welsh SO, Cronin FJ, et al. Number of days of food intake records required to estimate individual and group nutrient intakes with defined confidence. J Nutr 1987;117:1638-41. 